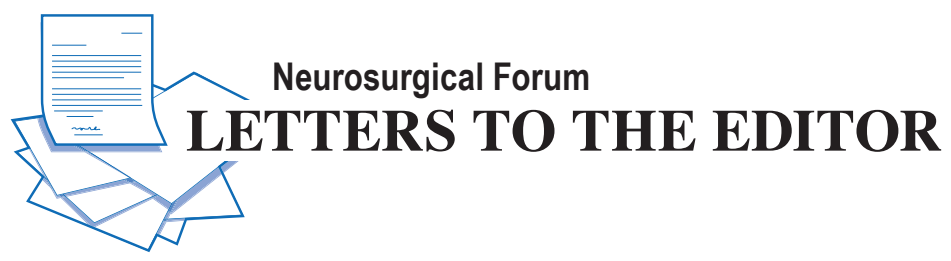

\section{Lateral sinus thrombosis: antithrombotic therapy and the issue of management of cerebellar swelling}

TO THE EDITOR: We read with great interest the article by Apra et al. ${ }^{1}$ (Apra C, Kotbi O, Turc G, et al: Presentation and management of lateral sinus thrombosis following posterior fossa surgery. $J$ Neurosurg [epub ahead of print February 26, 2016. DOI: 10.3171/2015.11. JNS151881]) concerning sinus thrombosis after posterior fossa surgery. The authors retrospectively analyzed 180 posterior fossa surgical procedures for removal of different space-occupying lesions at a single institution between January 2008 and May 2014; they focused their study on cases of postoperative lateral sinus thrombosis (LST). The authors clearly elucidated the incidence and risk factors associated with this entity and properly concluded that well-tolerated postoperative LST in asymptomatic patients should be managed conservatively.

Management of sinus thrombosis following posterior fossa surgery is a topic of debate in the neurosurgical literature, and clear therapeutic guidelines do not exist. Therefore, we would really like to thank the authors for their contribution and for having shared their experience in the treatment of asymptomatic patients. What appears to be more intriguing is how to treat those rare cases of patients who become symptomatic and exhibit clinical worsening due to cerebellar swelling. Indeed, sinus thrombosis associated with acute cerebellar swelling represents a life-threatening condition that may induce rapid neurological deterioration that is reversible only by prompt and adequate treatment, such as emergency posterior fossa decompressive craniectomy. ${ }^{2,5,7}$

Our contribution to the discussion comes from the following illustrative case. A 55-year-old woman presented to our institution with a large left petroclival meningioma (Fig. 1A) causing brainstem compression. Surgery aimed at subtotal resection, and optimal brainstem decompression was performed through a retrosigmoid approach. In the early postoperative course, the patient did not present any new neurological deficit, apart from slight diplopia due to manipulation of cranial nerves IV and VI. LST was ruled out on MR images obtained on postoperative Day 1 (Fig. 1B). However, on the 2nd postoperative day, the patient developed headache, nausea, and photophobia, and her level of consciousness had deteriorated since the previous day; hydrocephalus was diagnosed, and a ventriculoperitoneal shunt was immediately placed. Despite shunting, she did not regain her neurological status of the first postoperative day and remained in a stuporous condition that was progressively worsening. New MRI and CT scans disclosed a left LST and severe cerebellar swelling with effacement of the fourth ventricle and basal cisterns (Fig. $1 \mathrm{C}-\mathrm{E})$. A wide posterior fossa decompressive craniectomy with brainstem decompression was immediately performed, allowing for reappearance of the fourth ventricle and basal cisterns (Fig. 1F); this procedure stopped neurological deterioration and likely saved the patient's life.

Apra et al. confirmed the general feeling that antithrombotic therapy may increase hemorrhagic risk. In fact, they found that the incidence of surgical complications, secondlook surgeries, and surgical bed hemorrhage was higher in the group of patients who received antithrombotic therapy than in those who did not $(56.2 \%$ vs $27 \%, 37.5 \%$ vs $13.4 \%$, and $18.8 \%$ vs $10.4 \%$, respectively). ${ }^{1}$ Conservative management and a strict follow-up therefore appear to be the best option for asymptomatic patients.

However, in our view, attention should be focused on the symptomatic group. In fact, in symptomatic patients, the issue of emergency cerebellar decompression related to acute cerebellar swelling requires further debate. We believe that the real challenge for the surgeon is to recognize among the population of patients with postoperative LST those few who will develop cerebellar swelling, given the fact that posterior fossa sinus thrombosis and acute cerebellar swelling may amount to a vicious circle, where one condition leads to another, and vice versa. In such cases, the development of a posterior fossa "compartment syndrome" leads to a sudden local increase in intracranial pressure, ${ }^{3,4,6}$ which should be properly managed with very early surgical decompression in order to prevent brainstem damage (Fig. 1C and E). The use and the effects of antithrombotic therapy in these critical patients deserve maximal attention and debate.

Cerebellar swelling after posterior fossa surgery may develop even in absence of LST; ${ }^{4}$ in fact, the exact percentage of patients with postoperative LST who will develop cerebellar swelling is unknown. Certainly, LST appears to be a significant risk factor. But, if this is the case, should we look for LST with an early and dedicated postopera- 

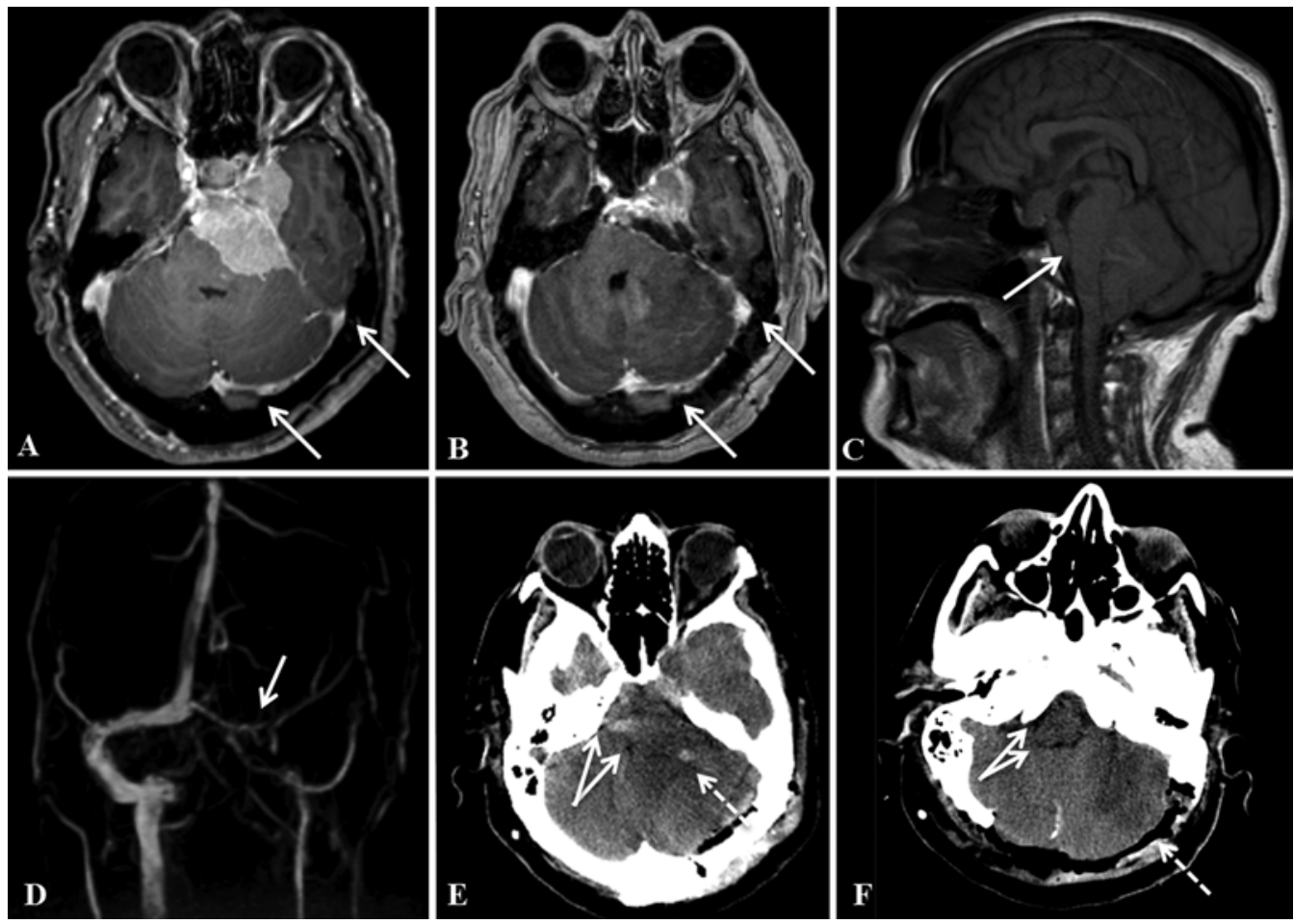

FIG. 1. A: Axial T1-weighted MR image with intravenous contrast administration showing a $50 \times 40 \times 50$-mm contrast-enhancing left petroclival and left cavernous sinus lesion; the left nondominant transverse and sigmoid sinuses are patent (arrows). B: Axial T1-weighted MR image with intravenous contrast administration obtained on postoperative Day 1, showing the results of resection with optimal brainstem decompression and residual tumor in the cavernous sinus; the left transverse and sigmoid sinuses are patent (arrows). C: Sagittal MR image obtained on postoperative Day 4, showing a cerebellar venous infarction causing initial herniation of the cerebellum through the foramen magnum and anterior displacement of the brainstem (arrow). D: Angio-MR reconstruction obtained on postoperative Day 4, showing complete occlusion of the left transverse and sigmoid sinuses (arrow) compared with the normal contralateral sinuses. E: Axial CT scan obtained on postoperative Day 4, showing cerebellar swelling, almost complete effacement of the basal cisterns and the fourth ventricle (arrows), and a hyperdense spot within the cerebellum (dotted arrow) due to an initial hemorrhagic infarction (perhaps due to LST). F: Axial CT scan obtained after the posterior fossa decompressive craniectomy, showing an impressive cerebellar herniation through the craniectomy (dotted arrow) and the renewed presence of the previously effaced fourth ventricle and basal cisterns (arrows).

tive MRI sequence in all patients during the postoperative course? And, if yes, when, given that thrombosis may not be evident on MRI study obtained on the 1st postoperative day as happened in our case? Should we base this diagnosis on MRI findings only or should we consider any other examination?

Furthermore, if thrombosis is detected, should we immediately start high-dosage antithrombotic treatment aiming at sinus recanalization or should we give the patient a "usual" low dose of enoxaparin (e.g., 4000 UI once per day) and wait to see what happens to the cerebellum? Apra et al. showed that high-dosage antithrombotic therapy required a long period of time for sinus recanalization ( $272 \pm 23$ days). Is it of paramount importance to start it early? Moreover, what is the gold-standard dose of enoxaparin for postoperative posterior fossa surgery?

Conversely, if the course of LST is not a significant predictor of cerebellar swelling, should we avoid high doses of antithrombotic drugs to prevent hemorrhagic complications? The rate of second-look surgery for hemorrhagic complications in the series by Apra et al. was almost 20\%; this seems much higher than our posterior fossa surgery series of the last 5 years or other series available in the literature, with an incidence around $5 \% .{ }^{4}$ Could this dif- ference be attributed to a different attitude toward the administration of antithrombotic therapy at our institution? Indeed, the safe/unsafe dose limit for antithrombotic therapy in neurosurgery is not clear.

Different attitudes and approaches to posterior fossa sinus thrombosis among high-volume centers highlights the importance of the topic addressed by Apra et al. and how posterior fossa surgery is still a "craft work" rather than a standardized procedure. Further studies involving many centers that share common protocols of postoperative imaging and management are required to further clarify these important issues.

Giacomo Bertolini, MD

Francesco Restelli, MD

Morgan Broggi, MD, PhD

Paolo Ferroli, MD

Fondazione IRCCS Istituto Neurologico Carlo Besta, Milano, Italy

\section{References}

1. Apra C, Kotbi O, Turc G, Corns R, Pagès M, SouillardScémama R, et al: Presentation and management of lateral sinus thrombosis following posterior fossa surgery. J 
Neurosurg [epub ahead of print February 26, 2016. DOI: 10.3171/2015.11.JNS151881]

2. Bertalanffy H: Avoidance of postoperative acute cerebellar swelling after pineal tumor surgery. Acta Neurochir (Wien) 158:59-62, 2016

3. Broggi M, Restelli F, Acerbi F, Ferroli P: Postoperative acute cerebellar swelling after pineal surgery: pathogenesis and treatment. Acta Neurochir (Wien) 158:63-65, 2016

4. Dubey A, Sung WS, Shaya M, Patwardhan R, Willis B, Smith D, et al: Complications of posterior cranial fossa surgery - an institutional experience of 500 patients. Surg Neurol 72:369-375, 2009

5. Einhäupl K, Stam J, Bousser MG, De Bruijn SFTM, Ferro $\mathrm{JM}$, Martinelli I, et al: EFNS guideline on the treatment of cerebral venous and sinus thrombosis in adult patients. Eur J Neurol 17:1229-1235, 2010

6. Jakola AS, Bartek J, Mathiesen T: Venous complications in supracerebellar infratentorial approach. Acta Neurochir (Wien) 155:477-478, 2013

7. Weimar C: Diagnosis and treatment of cerebral venous and sinus thrombosis. Curr Neurol Neurosci Rep 14:417, 2014

\section{Disclosures}

The authors report no conflict of interest.

\section{Response}

We would like to thank Drs. Bertolini, Restelli, Broggi, and Ferroli for their comment on our study on the management of LST after posterior fossa surgery for removal of space-occupying lesions and for sharing their experience about a complex case of postoperative LST with lifethreatening cerebellar edema.

They address when to identify LST on postoperative MRI and how to treat symptomatic cases, which are still a matter of debate. Our data do not directly address these questions, as none of our patients under study were symptomatic. Symptomatic LST may manifest through hydrocephalus, cerebellar edema, ischemia, or hematoma. Should we treat LST or the complication in those cases? Although treatment-dose anticoagulant therapy is the treatment of spontaneous LST, even in case of concomitant hemorrhage, preventing the vicious circle of venous hyperpression, venous ischemia, and hydrocephalus, ${ }^{2}$ this does not automatically extend to postoperative LST, whose underlying mechanisms are different. Repeating brain MRI when clinical features change may help; appearance or progression of LST encourages treating it with treatment-dose anticoagulant therapy. Some teams even follow asymptomatic patients with LST and introduce treatmentdose anticoagulant therapy if LST extends, even if it is asymptomatic. In such a particular condition, Bertolini et al. highlight an important dilemma: immediately starting treatment-dose anticoagulant therapy aiming at sinus recanalization, or following a wait-and-see approach. Indeed, treatment-dose anticoagulant therapy cannot be expected to control life-threatening symptoms in a short period of time, and treating the complications seems most important in such cases. Because surgery cannot be performed under treatment-dose anticoagulant therapy, and because venous ischemia may transform into hemorrhage, which may in turn increase cerebellar edema, it seems appropriate to perform life-saving surgical decompression first. Bertolini et al. do not indicate their treatment-dose anticoagulant therapy, but the emergency surgery was key in saving the patient. Local thrombolysis is sometimes proposed in heparin-resistant spontaneous cerebral venous thrombosis, ${ }^{1}$ but it is not recommended postoperatively. Modern approaches to endovascular therapy (e.g., thrombo-aspiration, stent retriever) without thrombolysis may be promising options in postoperative symptomatic LST associated with cerebellar hemorrhage or ischemia without life-threatening mass effect. ${ }^{4}$

Bertolini et al. also noticed that our complication rates differed from others in the literature. ${ }^{3}$ We think that this difference can be explained by the variable accuracy of retrospective studies, the different follow-up modalities, the definition of the complication criteria, and the inclusion criteria: the surgical procedures vary from one study to the other (with Chiari decompression not included in our series, for instance), as do the population characteristics.

Overall, symptomatic postoperative LST is extremely rare. While preventing LST is documented, the treatment is not standardized. We acknowledge that further prospective multicentric studies are definitely necessary to define the best therapy.

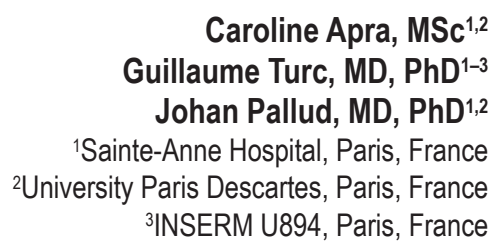

\section{References}

1. Calvet D, Bracard S, Mas JL: [Treatment of arterial and venous brain ischemia. Experts' recommendations: stroke management in the intensive care unit.] Rev Neurol (Paris) 168:512-521, 2012 (Fr)

2. Coutinho J, de Bruijn SF, Deveber G, Stam J: Anticoagulation for cerebral venous sinus thrombosis. Cochrane Database Syst Rev 8:CD002005, 2011

3. Dubey A, Sung WS, Shaya M, Patwardhan R, Willis B, Smith D, et al: Complications of posterior cranial fossa surgery-an institutional experience of 500 patients. Surg Neurol 72:369-375, 2009

4. Mokin M, Lopes DK, Binning MJ, Veznedaroglu E, Liebman KM, Arthur AS, et al: Endovascular treatment of cerebral venous thrombosis: contemporary multicenter experience. Interv Neuroradiol 21:520-526, 2015

\section{INCLUDE WHEN CITING}

Published online October 21, 2016; DOI: 10.3171/2016.7.JNS161729.

CAANS, 2017

\section{Comparison between surgery and Gamma Knife radiosurgery for brain AVMs}

TO THE EDITOR: We read with interest the article by Starke et al..$^{10}$ (Starke RM, Kano H, Ding D, et al: Stereotactic radiosurgery for cerebral arteriovenous malforma- 
tions: evaluation of long-term outcomes in a multicenter cohort. J Neurosurg [epub ahead of print March 4, 2016. DOI: $10.3171 / 2015.9 . J N S 151311])$.

Starke and colleagues are to be congratulated on producing a grading system (the Virginia Radiosurgery AVM Scale [VRAS]) for estimating the likelihood of favorable outcomes (defined as arteriovenous malformation [AVM] obliteration without postradiosurgery hemorrhage or permanent Gamma Knife radiosurgery [GKRS]-associated symptoms) following radiosurgery. ${ }^{11}$ The scale is simple to apply $\left(0,1\right.$, and 2 points for brain AVM volumes $<2 \mathrm{~cm}^{3}$, $2-4 \mathrm{~cm}^{3}$, and $>4 \mathrm{~cm}^{3}$, respectively, with additional points for a history of hemorrhage or location in eloquent brain as defined by the Spetzler-Martin grading system ${ }^{8}$ ) and was validated to be more discriminative than previous grading systems for GKRS. ${ }^{10,11}$ From the combined cohort series of 2236 cases retrospectively analyzed by Starke et al., approximately $81 \%, 75 \%, 65 \%, 47 \%$, and $41 \%$ of the patients with VRAS scores of $0,2,3$, and 4 , respectively, experienced a favorable outcome (at a mean of 7 years following treatment). ${ }^{10}$ The spread of outcomes and ease of application supports the wide adoption of this scale. This will be useful for clinicians in advising patients of the likelihood of favorable outcomes following radiosurgery.

However, greater assistance for patients with newly diagnosed brain AVMs is provided by utilizing the VRAS a step earlier, that is when making a recommendation of what type of treatment is best based upon the likelihood of providing the most favorable outcome. The highest quality evidence assisting comparison of outcomes from various treatments is from a randomized control trial (RCT). A controversial RCT, ARUBA, compared treatment versus no treatment for unruptured brain AVMs. ${ }^{6}$ In ARUBA, the wide range of mixtures of treatment methods and the small number of cases makes comparison of different treatment modalities impossible. ${ }^{6}$ Furthermore, the unfavorable results of treatment may reflect the outcomes expected from embolization rather than surgery or radiosurgery. ${ }^{1}$ In the absence of guidelines derived from an RCT, cohort studies currently remain an important source of informing best evidence-based medical practice. ${ }^{5}$ Therefore, a cohort series of the size reported by Starke and colleagues cannot be ignored in trying to inform opinion as to the recommended best practice. In addition to favorable outcomes stratified by VRAS score, Starke and colleagues have provided the results of favorable outcome per Spetzler-Martin grade in their Fig. 2 and the distribution of cases according to the Spetzler-Martin grade in their Table 2. ${ }^{10}$ We have estimated the number of cases from this information to derive a $95 \%$ confidence interval by the modified Wald technique (Table 1). This allows for comparison with surgical series graded similarly.

There are many unavoidable methodological reasons limiting comparison with cohort surgical series. For the series of Starke and colleagues, ${ }^{10}$ these include 1) not including events between diagnosis and treatment (e.g., prior treatment by embolization, radiotherapy, or surgery in $22 \%, 9 \%$, and $5 \%$ of cases, respectively), 2) not including cases with intention to treat by radiosurgery in which the patient did not progress to radiosurgery due to complications, 3) exclusion of cases deemed unsuitable for radiosurgery, and 4) referral bias.
These limitations can be equally applied to most cohort surgical series. In addition, length of follow-up after surgery is relatively short in most such series. However, we have attempted to analyze the prospectively collected surgical series of the corresponding author in a way that corrects for some of these biases and these results have been reported on a number of occasions..$^{1,2,4,7}$ One of our biases of the surgical series that assists comparison is that surgery (with or without preoperative embolization) and conservative management were the only 2 management options generally considered by the senior author (M.K.M.) before a Gamma Knife unit recently became available in Australia. Therefore, reported results from surgery can be said to reflect the results from a non-cherry-picked group of patients. Furthermore, we have the data for cases that were not treated by surgery (including the reason that surgery was not recommended). These excluded cases can be incorporated into a sensitivity analysis, with the assumption that a complication would have occurred had the patients undergone surgery. These cases, and the reasons for their exclusion, have been previously reported. ${ }^{4,7}$ No patient with a Spetzler-Martin Grade I or II (Spetzler Ponce Class $^{9}$ [SPC] A) AVM was denied surgery because of perceived surgical risk, 6 of 205 patients with Spetzler-Martin Grade III (SPC B) AVMs were denied surgery because of perceived surgical risk, and 33 of 112 patients with Spetzler-Martin Grades 4 and 5 (SPC C) AVMs were denied surgery because of perceived surgical risk. ${ }^{4,7}$ Another of the corrected biases in our series accounted for in the analysis is failure to progress from embolization to surgery because of complications occurring as a consequence of embolization. These adverse outcomes were included in the reported series as surgical complications. In addition, the length of follow-up and robustness of obliteration

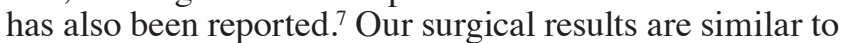
those of other reported cohort series and have been incorporated into pooled analyses. ${ }^{3,9}$ Therefore, it is reasonable to assume that our results may be generalizable to other comparable centers. We also believe that it is reasonable to compare our results with those obtained from Starke and colleagues' combined cohort.

To achieve this comparison, we have re-analyzed our previously reported cohort series ${ }^{4,7}$ with a comparable outcome definition (comparable to that used by Starke and colleagues) for "favorable outcome." We have interpreted "favorable outcome" in our surgical series to include patients free of brain AVMs, absence of brain AVM-related hemorrhage following surgery, and no complications from either embolization or surgery (including those complications from embolization that prevented the patient progressing to intended surgery) leading to a modified Rankin Scale score $>1$ at 12 months after surgery. We performed a sensitivity analysis to assign those cases in which surgery was denied because of an "unfavorable" perceived surgical risk. In order to obtain data for a comparative analysis, it was necessary to interpret Fig. 2 from the paper by Starke et al. to derive point estimates and combine the results with the data provided in their Table 2 to derive confidence intervals. This is therefore an approximation to the actual number of favorable outcomes per Spetzler-Martin grades (the actual number is not provided in the paper). The comparison of outcomes between 
TABLE 1. Rates of favorable outcome of surgical treatment of AVMs corrected for cases in which surgery was denied because of perceived surgical risk versus rates of favorable outcome of radiosurgery reported by Starke et al.*

\begin{tabular}{|c|c|c|c|c|}
\hline \multirow[b]{2}{*}{ Outcome } & \multicolumn{3}{|c|}{ From Korja et al. } & \multirow[b]{2}{*}{$\begin{array}{l}\text { Rate of "Favorable Outcome" in GKRS } \\
\text { Series of Starke et al. }(95 \% \mathrm{CI}) \S\end{array}$} \\
\hline & $\begin{array}{l}\text { No. of Pts w/ "Favorable } \\
\text { Outcome" of Op } \dagger\end{array}$ & $\begin{array}{c}\text { No. of Pts Denied Op Because } \\
\text { of Perceived Risk }\end{array}$ & $\begin{array}{l}\text { Corrected Rate of Favorable } \\
\text { Outcome }(95 \% \mathrm{Cl}) \ddagger\end{array}$ & \\
\hline SPC A & 357 of 363 & 0 & $98.4 \%(96.4-99.3 \%)$ & $68 \%(65-71 \%)$ \\
\hline SPC B & 156 of 199 & 6 & $76 \%(70-81 \%)$ & $60 \%(57-63 \%)$ \\
\hline SPC C & 45 of 79 & 33 & $40 \%(32-49 \%)$ & $36 \%(30-42 \%)$ \\
\hline Total & 558 of 641 & 39 & $82 \%(79-85 \%)$ & $61 \%(59-63 \%)$ \\
\hline Length of FU (mean) & $6 \mathrm{yrs}$ & NA & NA & 7 yrs \\
\hline
\end{tabular}

FU = follow-up; NA = not applicable; pts = patients.

* "Favorable outcome" for surgery defined as follows: patients free of brain AVM, no brain AVM-related hemorrhage following surgery, and no complications from either embolization or surgery (including those complications from embolization that prevented the patient progressing to intended surgery) leading to a modified Rankin Scale score at 12 months of greater than 1. "Favorable outcome" for radiosurgery defined as AVM obliteration without postradiosurgery hemorrhage or permanent GKRSassociated symptoms.

$\dagger$ Rate of favorable outcome (as defined above) for patients undergoing surgery reanalyzed from series reported by Korja et al. ${ }^{4}$

$\ddagger$ Corrected for patients denied surgery because of perceived risk.

$\S$ Approximated from Fig. 2 and Table 2 in Starke et al. ${ }^{10}$

our study and that of Starke and colleagues can be seen in Table 1. This comparison suggests that there is an advantage of surgery over radiosurgery for patients with SPC A and SPC B AVMs with respect to "favorable outcomes."

The VRAS is not only a useful tool to inform a patient undergoing radiosurgery as to the proportion of similar cases that will have a "favorable outcome," but it is also useful for comparing surgery with radiosurgery. With regard to "favorable outcome," surgery would appear to offer an advantage over radiosurgery for SPC A and B AVMs, with neither treatment providing comfort for patients with SPC C lesions. Of course, there are many factors on which a decision to recommend surgery versus radiosurgery can be based. These include the degree of deficit, the time to deficit, and comorbidities, to mention but a few. Many of the investigators who contributed to Starke and colleagues' series have previously reported that for SPC A brain AVMs, surgery is the preferred option. This recommendation is again emphasized with their reported outcomes. Even with the best VRAS grade of 0 , favorable outcomes were observed in only $81 \%$ of cases, ${ }^{10}$ a result that is better than outcomes of surgery for SPC B AVMs but not as good as outcomes of surgery for SPC A AVMs.

Another method of comparing surgery with radiosurgery involves adoption of the accountancy tool of costeffectiveness in which the cost or complications (incorporating patients denied surgery because of perceived surgical risk) are reported per effectively treated AVM. In such an analysis, we have previously reported that the cumulative risk of complications at 9 years after surgery in cases of effectively treated AVMs (complication-effectiveness analysis) is $1.4 \%, 24 \%$, and $63 \%$ for patients with SPC A, B, and C lesions, respectively. ${ }^{7}$ It will be interesting to see this method of analysis used for other treatment modalities. It is important to look at outcomes from a variety of perspectives (e.g., complication-effectiveness and "favorable" outcomes to name two) that facilitate comparison between treatments and that take into account differences in feasibility of treatment (i.e., analysis to incorporate those denied treatment by a modality of treatment because of perceived risk), obliteration rates, time to obliteration (and exposure to the continuing risk of hemorrhage), and complications.

\section{Michael Kerin Morgan, MD \\ Marcus Andrew Stoodley, PhD \\ John William Fuller, FRACS \\ Macquarie University, Sydney, Australia}

\section{References}

1. Bervini D, Morgan MK, Ritson EA, Heller G: Surgery for unruptured arteriovenous malformations of the brain is better than conservative management for selected cases: a prospective cohort study. J Neurosurg 121:878-890, 2014

2. Davidson AS, Morgan MK: How safe is arteriovenous malformation surgery? A prospective, observational study of surgery as first-line treatment for brain arteriovenous malformations. Neurosurgery 66:498-505, 2010

3. Kim H, Abla AA, Nelson J, McCulloch CE, Bervini D, Morgan MK, et al: Validation of the supplemented Spetzler-Martin Grading system for brain arteriovenous malformations in a multicenter cohort of 1009 surgical patients. J Neurosurg 76:25-33, 2015

4. Korja M, Bervini D, Assaad N, Morgan MK: The role of surgery in the management of brain arteriovenous malformations: a prospective cohort study. Stroke 45:3549-3555, 2014

5. Korja M, Hernesniemi J, Lawton MT, Spetzler RF, Morgan MK: Is cerebrovascular neurosurgery sacrificed on the altar of RCTs? Lancet 384:27-28, 2014

6. Mohr JP, Parides MK, Stapf C, Moquete E, Moy CS, Overby JR, et al: Medical management with or without interventional therapy for unruptured brain arteriovenous malformations (ARUBA): a multicenter, non-blinded, randomized trial. Lancet 383:614-621, 2014

7. Morgan MK, Wiedmann M, Assaad N, Heller GZ: Complication-effectiveness analysis for brain arteriovenous malformation surgery. A prospective cohort study. Neurosurgery 79:47-57, 2016

8. Spetzler RF, Martin NA: A proposed grading system for arteriovenous malformations. J Neurosurg 65:476-483, 1986

9. Spetzler RF, Ponce FA: A 3-tier classification of cerebral arteriovenous malformations. Clinical article. J Neurosurg 114:842-849, 2011 
10. Starke RM, Kano H, Ding D, Lee JYK, Mathieu D, Whitesell $\mathrm{J}$, et al: Stereotactic radiosurgery for cerebral arteriovenous malformations: evaluation of long-term outcomes in a multicenter cohort. J Neurosurg [epub ahead of print March 4, 2016. DOI: 10.3171/2015.9.JNS151311]

11. Starke RM, Yen CP, Ding D, Sheehan JP: A practical grading scale for predicting outcome after radiosurgery for arteriovenous malformations: analysis of 1012 treated patients. J Neurosurg 119: 981-987, 2013

\section{Disclosures}

The authors report no conflict of interest.

\section{Response}

We thank Dr. Morgan and his colleagues for their interest in our recent studies and their acknowledgement of the utility of the VRAS. ${ }^{6}$ In these studies, the 8 participating centers had a long history of microsurgery along with embolization of AVMs, and certainly many patients were treated with therapies other than radiosurgery during the more than 25 years in which these patients were accrued. Additionally, a number of patients were observed without intervention during this time frame. Although there has been much discussion regarding the treatment of unruptured AVMs, radiosurgery is a beneficial therapy for carefully selected patients. ${ }^{2,3,5} \mathrm{~A}$ direct comparison between radiosurgery and microsurgery as carried out by Morgan et al. is challenging due to inherent observed and unobserved differences in patient and AVM characteristics. Although a motor cortex AVM can be a Spetzler-Martin Grade II malformation, most would argue that the role of microsurgery in this instance is reduced due to the higher risk of associated neurological decline. Although surgery may be the preferable treatment for some patients, it is also associated with a number of unaccounted-for risks, including meningitis, venous thromboembolism, and increased risk of seizures and higher procedural costs (e.g., inpatient hospitalization) that are not associated with stereotactic radiosurgery. Additional elements that go into patient assessment and treatment selection are not easily accounted for by a simplified grading scale. Other potential limitations may further confound the analysis of Morgan and colleagues, including but not limited to selection, referral, attrition, funding, reporting, recall, and observer bias. ${ }^{1}$

We agree that although not initially derived to predict outcomes of patients undergoing surgery or embolization for the treatment of AVMs, the VRAS may have utility in these cases or in multimodal therapy planning, but this assertion requires further validation.

In the modern era of neurosurgery, it is important to move beyond single-center, retrospective studies if we hope to refine patient care algorithms in a meaningful way. Multicenter studies convey greater validity to a study's findings, and more sophisticated approaches such as propensity score matching are readily employed when statistical power is increased by accruing patients from multiple centers. The motivation to provide higher levels of evidence to the literature was one of the reasons for creating the International Gamma Knife Research Foundation (IGKRF). The IGKRF currently includes more than 30 member sites across 3 continents. Further multicenter studies on AVMs are forthcoming from our group, and others are expected to arise from the national SRS regis- try of the American Association of Neurological Surgeons and the American Society for Radiation Oncology. ${ }^{4}$

$$
\begin{array}{r}
\text { Robert M. Starke, MD, MSc } \\
\text { Jason P. Sheehan, MD, PhD } \\
\text { Gamma Knife Center, University of Virginia, Charlottesville, VA } \\
\text { Douglas Kondziolka, MD } \\
\text { New York University Langone Medical Center, New York, NY } \\
\text { L. Dade Lunsford, MD } \\
\text { University of Pittsburgh, Pittsburgh, PA }
\end{array}
$$

\section{References}

1. Bennett CM, Baird AA, Miller MB, Wolford GL: Neural correlates of interspecies perspective taking in the post-mortem Atlantic salmon: an argument for proper multiple comparisons correction. J Serendipitous Unexpected Results 1:1-5, 2010

2. Ding D, Starke RM, Kano H, Mathieu D, Huang P, Kondziolka D, et al: Radiosurgery for cerebral arteriovenous malformations in A Randomized Trial of Unruptured Brain Arteriovenous Malformations (ARUBA)-eligible patients: a multicenter study. Stroke 47:342-349, 2016

3. Mohr JP, Parides MK, Stapf C, Moquete E, Moy CS, Overbey JR, et al: Medical management with or without interventional therapy for unruptured brain arteriovenous malformations (ARUBA): a multicentre, non-blinded, randomised trial. Lancet 383:614-621, 2014

4. Sheehan JP, Kavanagh BD, Asher A, Harbaugh RE: Inception of a national multidisciplinary registry for stereotactic radiosurgery. J Neurosurg 124:155-162, 2016

5. Starke RM, Sheehan JP, Ding D, Liu KC, Kondziolka D, Crowley RW, et al: Conservative management or intervention for unruptured brain arteriovenous malformations. World Neurosurg 82:e668-e669, 2014

6. Starke RM, Yen CP, Ding D, Sheehan JP: A practical grading scale for predicting outcome after radiosurgery for arteriovenous malformations: analysis of 1012 treated patients. J Neurosurg 119:981-987, 2013

\section{Disclosures}

L. Dade Lunsford reports a consultant relationship with and stock ownership in Elekta $\mathrm{AB}$ and a consultant relationship with Insightec, DSMB.

INCLUDE WHEN CITING

Published online July 29, 2016; DOI: 10.3171/2016.3.JNS16634.

(cAANS, 2017

\section{Simpson grading system}

TO THE EDITOR: I read the recent article by Nanda and colleagues ${ }^{4}$ regarding the relevance of the Simpson grading system in modern neurosurgery with great interest (Nanda A, Bir SC, Maiti TK, et al: Relevance of Simpson grading system and recurrence-free survival after surgery for World Health Organization Grade I meningioma. J Neurosurg [epub ahead of print April 8, 2016. DOI: 10.3171/2016.1.JNS151842]). The authors reviewed more than 20 years of surgical records, and analyzed the cases of 458 patients with meningioma who had undergone re- 
sective surgery. Of the total cases, 330 were included in an analysis of the Simpson grading system's prognostic capability, particularly its ability to predict recurrencefree survival (RFS). The overall tumor recurrence rates for Simpson Grades I, II, III, and IV were 5\%, 22\%, 31\%, and $35 \%$, respectively, with the RFS after Grade I and II resections being significantly longer than after Grade III and IV resections. The authors concluded that the significance of the Simpson grading system is well established, and that improvement of the Simpson grade remains the fundamental objective in most meningioma cases.

The conclusions of Nanda and colleagues are consistent with other recent studies that reappraised the significance of the Simpson grading system in the context of modern neurosurgical techniques and radiological examinations, and found it to have endured. ${ }^{1,2,4}$ I fully agree with the assertion in these studies that achieving high resection rates in meningioma surgery contributes to the minimization of the recurrence rate and the long-term maintenance of neurological functions. However, I am constantly puzzled by the kind of disclaimer that always seems to follow this type of strong statement, such as "whenever such a strategy appears reasonably safe" or "surgery should be tailored to each patient according to the risks and surgical morbidity," because no clarifications are provided regarding how to appropriately weigh the risks and benefits of aggressive resections in cases of histologically benign tumors, in order to balance safety and efficacy.

Whereas the above-mentioned studies emphasized the fadeless significance of the Simpson grading system, some other studies have reported that the predictive power of the system for recurrence has been diluted in modern neurosurgery. ${ }^{3,5,6}$ Interestingly, the number of tumors analyzed in these studies ranges from 183 to 373 , which is a smaller sample size than that of most studies in which tumor grade was found to be a significant predictor of RFS (range 330-714 tumors). Although this could be written off as unimportant or a coincidence, I believe it may be the key to unlocking this controversy. In the context of retrospective studies, any correlation will eventually reach statistical significance if enough samples are included. Although no one would dispute the importance of resecting meningiomas whenever possible, statistical evidence supporting this strategy cannot possibly come out of only a few hundred patients. It probably takes longer than 10 years for large neurosurgical institutions to treat several hundred or more patients with meningiomas. Accordingly, not many neurosurgeons will perform a sufficient number of meningioma surgeries in their career to produce statistically significant correlations between RFS and tumor grade. We know that a certain percentage of WHO Grade I meningiomas grow very little in the years following surgery. Therefore, for some tumors, taking a significant risk in the first surgery may be unwarranted. Ideally, an examination would be performed intraoperatively to assess the biological features of each tumor and to determine the risk of recurrence; however, such a procedure is not presently established.

To sum up, statistical significance is not unequivocal, and surgeons should make every effort to tailor the surgical strategy for each patient. I agree that an aggressive resection strategy is usually the best policy in meningioma surgery, as Nanda and colleagues have demonstrated in their commendable study. However, we should be aware that the recent "reappraisal of the Simpson grading system" is substantiated by the data of several hundred patients, rather than by applying it to all cases.

Soichi Oya, MD, PhD

Saitama Medical Center, Saitama Medical University, Saitama, Japan

\section{References}

1. Gousias K, Schramm J, Simon M: The Simpson grading revisited: aggressive surgery and its place in modern meningioma management. J Neurosurg [epub ahead of print January 29, 2016; DOI: 10.3171/2015.9.JNS15754]

2. Hasseleid BF, Meling TR, Rønning P, Scheie D, Helseth E: Surgery for convexity meningioma: Simpson Grade I resection as the goal. J Neurosurg 117:999-1006, 2012

3. Heald JB, Carroll TA, Mair RJ: Simpson grade: an opportunity to reassess the need for complete resection of meningiomas. Acta Neurochir (Wien) 156:383-388, 2014

4. Nanda A, Bir SC, Maiti TK, Konar SK, Missios S, Guthikonda B: Relevance of Simpson grading system and recurrencefree survival after surgery for World Health Organization Grade I meningioma. J Neurosurg [epub ahead of print April 8, 2016; DOI: 10.3171/2016.1.JNS151842]

5. Oya S, Kawai K, Nakatomi H, Saito N: Significance of Simpson grading system in modern meningioma surgery: integration of the grade with MIB-1 labeling index as a key to predict the recurrence of WHO Grade I meningiomas. J Neurosurg 117:121-128, 2012

6. Sughrue ME, Kane AJ, Shangari G, Rutkowski MJ, McDermott MW, Berger MS, et al: The relevance of Simpson Grade I and II resection in modern neurosurgical treatment of World Health Organization Grade I meningiomas. J Neurosurg 113:1029-1035, 2010

\section{Disclosures}

The author reports no conflict of interest.

\section{Response}

Thank you for the opportunity to respond to Dr. Oya's letter.

We appreciate Dr. Oya's feedback about our article, "Relevance of Simpson grading system and recurrencefree survival after surgery for World Health Organization Grade I meningioma." He has noted one interesting finding: that the predictive power of Simpson grading is more evident for a larger series than for a relatively smaller one. He also postulated that a neurosurgeon in a smaller setup may not treat enough cases to evaluate the significance of Simpson grading in his or her own practice. We are in complete agreement with this. Furthermore, the patients need to be followed up judiciously over at least 5-7 years to estimate the RFS. With advancements in the knowledge of the genetic pathway related to meningiomas, it is very well possible to find the subset of these tumors (even within WHO Grade I) that are more likely to recur. Another potential limitation to studies of the relevance of Simpson grading in the future is the increasing use of radiosurgery for residual tumor in the immediate postoperative period. Surgeons are inclined to subject the patient to radiosurgery, even one with small residual tumor.

To weigh the risks and benefits of aggressive resection 
is also critical, especially for the surgically challenging locations, such as petroclival or cavernous sinus meningiomas. Maximal subtotal resection followed by radiosurgery will address the patient's concern, but may leave the neurosurgeon disappointed at not achieving a Simpson Grade I or II resection. Surgical wisdom is important here along with technical finesse, to understand "where to stop." In The Iliad, Achilles must choose between kleos or nostosglory or a safe return home. In the end, patient satisfaction and functional outcomes are more important than tumorfree postoperative MR images.

Anil Nanda, MD, MPH Tanmoy K. Maiti, MD, MCh

Shyamal C. Bir, MD, PhD

LSU Health-Shreveport, Shreveport, LA

\section{INCLUDE WHEN CITING}

Published online September 23, 2016; DOI: 10.3171/2016.5.JNS161302.

oAANS, 2017

\section{The bispectral index is a dimensionless number}

TO THE EDITOR: In their recent article, Ohtaki et al. ${ }^{9}$ (Ohtaki S, Akiyama Y, Kanno A, et al: The influence of depth of anesthesia on motor evoked potential response during awake craniotomy. J Neurosurg [epub ahead of print March 4, 2016. DOI: 10.3171/2015.11.JNS151291]) indicated that the bispectral index (BIS) shows a positive correlation with motor evoked potential (MEP) amplitude, whereas it has a negative correlation with MEP latency under propofol monoanesthesia. They also mentioned that they are the first to demonstrate that MEP latency decreases in accordance with increasing BIS value. Although the authors performed an excellent study, even discussing several study limitations, we have some reservations about the methodology and data analysis in the study.

The BIS is a kind of processed electroencephalography (EEG) monitor whose index value is calculated based on a large database of high-fidelity EEG activity using a proprietary algorithm. ${ }^{5,11}$ Because EEG is a complex signal representing brain electrical activity, EEG waveforms show different patterns according to montages. ${ }^{13}$ As Glass et al. reported, using frontal montage is one of the inclusion criteria for BIS development. ${ }^{3}$ Frontal EEG is considered capable of reflecting a gradual change in the anesthetic effect by using a combination of EEG amplitude, frequency, variability, topography, and frontal electromyography (EMG) activity. In the current study, Ohtaki et al. adopted a unique montage to assess the depth of anesthesia; a BIS self-adhesive EEG electrode strip was positioned from the contra- and ipsilateral nasal bones to the ipsilateral zygomatic bone. ${ }^{9}$ Actually, there is another report in which the authors used an occipital montage for BIS monitoring, ${ }^{12}$ however, as far as BIS heavily relies on frontal EEG patterns for its mathematical analysis, there is no guaranty that BIS-EEG from nonfrontal montages truly reflects the depth of anesthesia. Homology of raw EEG waveforms between frontal and nonfrontal montages should be validated when a nonfrontal montage is required for intraoperative BIS recording.

There are a number of limiting and confounding factors associated with BIS monitoring. Since the electrode array is located over the facial muscles, BIS values are elevated by sources of high-frequency artifacts, especially facial muscle activities. Unless removed by filters, this EMG activity can be a significant contribution to the recording and can produce significant errors in the index values. Alternatively, EMG activity can be a sign of impending arousal or inadequate anesthesia. ${ }^{10}$ Furthermore, external signals with the proper frequency content, such as electrocautery, pacemakers, endoscopic shavers, and warming devices, can generate erroneous indices. ${ }^{1}$ Ideally, the raw EEG signals are visually inspected by a physician who has enough experience to distinguish artifacts. Otherwise, BIS values with high signal quality index (SQI) should be solely selected for further analysis. Therefore, it is highly suspected that the BIS values collected in the present study do not accurately correlate with the hypnotic state because the authors did not determine cutoff levels of BIS-EMG or BIS-SQI. ${ }^{9}$ In particular, facial muscle activities may have contributed to higher BIS levels among the present population because the patients were asked to respond to intraoperative tasks during awake craniotomy. Additionally, we should remember that the relationship between BIS value and measured propofol concentration is nonlinear. There is a wide range of propofol plasma concentration from 1 to $10 \mu \mathrm{g} / \mathrm{ml}$ when the BIS indicates an adequate level of anesthesia as its value of $50 .^{3}$

It is notable that MEP latency decreased in accordance with increases in the BIS value in the current series, ${ }^{9}$ although there are several reports indicating that MEP latency did not significantly change following the administration of intravenous agents. ${ }^{4,6,8}$ In the pyramidal tract, there are only 2 synaptic connections; one is between the upper motor neuron and the alpha motor neuron, and the other is located in the neuromuscular junction. Thus, it is theoretically less affected by prolonged MEP latency due to anesthetics. It was suggested that the majority of anesthetic effects are amplitude changes in evoked responses with smaller changes in latency because the targets of general anesthetics primarily affect synaptic function rather than neuronal conduction. ${ }^{2}$ In propofol anesthesia, transcranial MEPs can usually still be recorded at the EEG burstsuppression level, though their amplitude is lower than that at lighter sedation levels.? The current study conducted by Ohtaki et al. ${ }^{9}$ is really interesting, so we ask the authors, by what mechanism does MEP latency increase when BIS numbers are lower?

Many types of intraoperative monitoring devices are available now. By understanding the physiological and pharmacological bases of anesthesia and monitoring, the operating room staff can considerably improve patient safety when acting as a team.

\section{Kotoe Kamata, MD, $\mathrm{PhD}^{1}$ Satoshi Hagihira, MD, $\mathrm{PhD}^{2}$ Takashi Maruyama, MD, $\mathrm{PhD}^{3,4}$}




\section{Yoshihiro Muragaki, $\mathrm{MD}, \mathrm{PhD}^{3,4}$ Makoto Ozaki, MD, PhD'}

${ }^{1}$ Tokyo Women's Medical University, Tokyo, Japan

${ }^{2}$ Surgical Center, Osaka Prefectural Osaka General Medical Center,

Osaka, Japan

${ }^{3}$ Neurological Institute, Tokyo Women's Medical University, Tokyo, Japan

${ }^{4}$ Faculty of Advanced Techno-Surgery, Institute of Advanced Biomedical Engineering and Science, Tokyo Women's Medical University,

Tokyo, Japan

\section{References}

1. Dahaba AA: Different conditions that could result in the bispectral index indicating an incorrect hypnotic state. Anesth Analg 101:765-773, 2005

2. Franks NP, Lieb WR: Which molecular targets are most relevant to general anaesthesia? Toxicol Lett 100-101:1-8, 1998

3. Glass PS, Bloom M, Kearse L, Rosow C, Sebel P, Manberg $\mathrm{P}$ : Bispectral analysis measures sedation and memory effects of propofol, midazolam, isoflurane, and alfentanil in healthy volunteers. Anesthesiology 86:836-847, 1997

4. Hargreaves SJ, Watt JWH: Intravenous anaesthesia and repetitive transcranial magnetic stimulation monitoring in spinal column surgery. Br J Anaesth 94:70-73, 2005

5. Johansen JW, Sebel PS: Development and clinical application of electroencephalographic bispectrum monitoring. Anesthesiology 93:1336-1344, 2000

6. Kalkman CJ, Drummond JC, Ribberink AA, Patel PM, Sano T, Bickford RG: Effects of propofol, etomidate, midazolam, and fentanyl on motor evoked responses to transcranial electrical or magnetic stimulation in humans. Anesthesiology 76:502-509, 1992

7. MacDonald DB, Al Zayed Z, Stigsby B: Tibial somatosensory evoked potential intraoperative monitoring: recommendations based on signal to noise ratio analysis of popliteal fossa, optimized P37, standard P37, and P31 potentials. Clin Neurophysiol 116:1858-1869, 2005

8. Nathan N, Tabaraud F, Lacroix F, Mouliès D, Viviand X, Lansade A, et al: Influence of propofol concentrations on multipulse transcranial motor evoked potentials. Br J Anaesth 91:493-497, 2003

9. Ohtaki S, Akiyama Y, Kanno A, Noshiro S, Hayase T, Yamakage M, et al: The influence of depth of anesthesia on motor evoked potential response during awake craniotomy. J Neurosurg [epub ahead of print March 4, 2016. DOI: 10.3171/2015.11.JNS151291]

10. Paloheimo M, Edmonds HL Jr, Wirtavuori K, Tammisto T: Assessment of anaesthetic adequacy with upper facial and abdominal wall EMG. Eur J Anaesthesiol 6:111-119, 1989

11. Rampil IJ: A primer for EEG signal processing in anesthesia. Anesthesiology 89:980-1002, 1998

12. Shiraishi T, Uchino H, Sagara T, Ishii N: A comparison of frontal and occipital bispectral index values obtained during neurosurgical procedures. Anesth Analg 98:1773-1775, 2004

13. Sigl JC, Chamoun NG: An introduction to bispectral analysis for the electroencephalogram. J Clin Monit 10:392-404, 1994

\section{Disclosures}

The authors report no conflict of interest. tions and comments regarding our work. They have asked 2 questions: 1) Do the BIS values in our study accurately correlate with the depth of anesthesia? 2) By what mechanism does MEP latency increase when the BIS number is lower?

As they noted, EMG signals are received from facial muscles as the level of arousal increases, and a time lag occurs as new BIS values are calculated (Aspect Medical Systems Inc.: Technology overview: bispectral index, 1997). Thus, there are situations in which the BIS does not accurately reflect the depth of anesthesia, and this fact has been mentioned as one of the limitations of our study. The BIS varies dramatically at values of up to $70-90$ in particular. In the 28 patients we studied, we examined variability in the mean amplitude and latency of the MEP, but those changes were not linear. This may have precluded assessment of the depth of anesthesia with the BIS in real time. However, when patients were in an awake state with a BIS value of 90 or higher, locomotion and a response to instructions were evident, indicating that the effects of propofol were minimized. Compared to patients under general anesthesia with a BIS value below 60, patients who were in the awake state with a BIS value of 90 or higher had a significantly higher MEP amplitude and a significantly shorter MEP latency and they had consistent MEP waveforms. Thus, a BIS value of 90 or higher remains a key method of assessing the MEP during arousal. A rise in or flattening of the amplitude of the MEP with the same intensity of stimulation results in more accurate assessment of motor function.

Propofol blocks $\alpha$-motor neurons in the anterior horn of the spinal cord, so stimulation with trains-of-five pulses during measurement of the MEP in an individual under general anesthesia causes temporal summation of the inputs to the $\alpha$-motor neurons, bringing anterior horn cells in the spinal cord to their firing threshold and eliciting an evoked potential. As the level of arousal increases (that is, as the level of propofol in the blood decreases), the blocking of motor neurons diminishes and stimulation with trains of fewer than 5 pulses can bring anterior horn cells to their firing threshold, as our study revealed. The latency of the MEP shortens since anterior horn cells fire with the very first or second pulse rather than with the fifth pulse. Propofol is known to block corticocortical projection neurons, and this may be another mechanism by which it affects the latency of the MEP.

Intraoperative electrophysiological evaluation such as MEP is critical to determine the direction of neurosurgery: for example, extension of tumor removal and reclipping the cerebral aneurysm. Since both muscle MEP and BIS monitoring are quantitative but general assessments for neurosurgery, we tried to verify the real relationship between these data and to make a clinically ideal judgment.

We hope our responses have sufficiently answered the questions we received. We would be happy to answer in greater detail if necessary.

\section{Response}

We thank Dr. Kamata and colleagues for their ques- 
INCLUDE WHEN CITING

Published online July 1, 2016; DOI: 10.3171/2016.3.JNS16774.

cAANS, 2017

\section{Cortical spreading depression}

TO THE EDITOR: We read with great interest the recently published article by Carlson et al. ${ }^{1}$ (Carlson AP, Shuttleworth CW, Mead B, et al: Cortical spreading depression occurs during elective neurosurgical procedures. J Neurosurg [epub ahead of print March 11, 2016. DOI: 10.3171/2015.11.JNS151871]), in which they reported the first spreading depolarizations (SDs) using intraoperative electrocorticography (iECoG). They reported 2 definite SDs and 8 more suspected SDs among 8 patients, using a DC amplifier. They also reported detecting 0 SDs among 3 patients using an AC amplifier. They described the advantages of the DC recording technique to detect intraoperative SDs; the results of deliberately seeking SDs intraoperatively are promising, since iECoG has been frequently used in neurosurgical centers for the last 5 decades for the following purposes: 1) to locate the irritative zone, 2) to map out cortical functions, and 3) to predict the success of surgery for epilepsy. The relevance that the SDs could have in this scenario is completely unknown. ${ }^{3,4}$

The article by Carlson et al. ${ }^{1}$ motivated us to investigate SDs in patient recordings acquired at the National Institute of Neurology and Neurosurgery in Mexico City, Mexico. We used the methodology described by the authors and by the COSBID (Co-Operative Studies on Brain Injury Depolarizations) group when looking for SDs. ${ }^{2}$ We retrospectively analyzed $81 \mathrm{iECoG}$ recordings from 68 patients with refractory epilepsy due to hippocampal sclerosis, which required tailored anterior temporal lobectomy combined with amygdalohippocampectomy.

The duration of the analyzed recordings is within a range of 35-50 minutes. The $\mathrm{iECoG}$ signals were recorded with a 64-channel digital AC amplifier (BE Plus, EBNeuro), typically using grids of 20 or 24 contacts $(5 \times 4$ or 6 $\times 4$ contacts with $1-\mathrm{cm}$ spacing [Integra]). ${ }^{4}$ The manufacturer indicates that this amplifier is capable of recording in "open band" mode, only limited by the hardware band pass (analog) filters used to prevent anti-aliasing.

Nevertheless, in our meticulous identification attempts (E.S. and A.O.R.), we found no SDs. Considering our analysis and the previous experiences of the COSBID group, ${ }^{2}$ we arrived at the conclusion that, for a prospective analysis, a DC recording technique with a theoretical filter of 0 $\mathrm{Hz}$ is essential.

Therefore, we are encouraged to acquire a DC amplifier to effectively detect SDs and to better understand these phenomena associated with neurosurgical procedures.

Daniel San-Juan, MD Andres Jaramillo-Gonzalez, MSc Roberto Diaz-Peregrino, MD

National Institute of Neurology and Neurosurgery, Mexico City, Mexico
Arturo Olivares Rivera, MD

Edgar Santos, MD

Heidelberg University Hospital, Heidelberg, Germany

\section{References}

1. Carlson AP, Shuttleworth CW, Mead B, Burlbaw B, Krasberg $\mathrm{M}$, Yonas H: Cortical spreading depression occurs during elective neurosurgical procedures. J Neurosurg [epub ahead of print March 11, 2016. DOI: 10.3171/2015.11.JNS151871]

2. Dreier JP, Fabricius M, Ayata C, Sakowitz OW, Shuttleworth $\mathrm{CW}$, Dohmen C, et al: Recording, analysis, and interpretation of spreading depolarizations in neurointensive care: review and recommendations of the COSBID research group. $\mathbf{J}$ Cereb Blood Flow Metab [epub ahead of print], 2016

3. Kuruvilla A, Flink R: Intraoperative electrocorticography in epilepsy surgery: useful or not? Seizure 12:577-584, 2003

4. San-Juan D, Díaz-Nuñez IC, Ojeda-Baldéz M, Barajas-Juárez VA, González-Hernández I, Alonso-Vanegas M, et al: Utility of electrocorticography in the surgical treatment of cavernomas presenting with pharmacoresistant epilepsy. Epileptic Disord 16:245-260, 2014

\section{Disclosures}

The authors report no conflict of interest.

\section{Response}

We appreciate the observations from Dr. Santos and colleagues regarding our recent article. Their very experienced team analyzed an extensive amount of $\mathrm{iECoG}$ data for signs of cortical SDs and were not able to identify any events when using a more standard AC amplifier setup. These kinds of rigorous negative analyses are critical to support the hypothesis that the reason that the events have not yet been widely identified in the vast $\mathrm{iECoG}$ experience is because of technical limitations rather than the true absence of events. We think that the use of the true full-spectrum DC amplifier has been very useful in the ICU environment and has facilitated identification of the hallmark DC shifts of SD even by novices with brief training. The slow potential changes used in $\mathrm{AC}$ recordings in both our ICU and OR experience are much more complex to interpret. ${ }^{1}$

We thank the authors for this interesting and useful observation and look forward to their future studies.

Andrew P. Carlson, MD, MSCR

C. William Shuttleworth, PhD

University of New Mexico School of Medicine, Albuquerque, NM

\section{References}

1. Hartings JA, Li C, Hinzman JM, Shuttleworth CW, Ernst GL, Dreier JP, et al: Direct current electrocorticography for clinical neuromonitoring of spreading depolarizations. J Cereb Blood Flow Metab [epub ahead of print], 2016 